\title{
ERROS IDENTIFICADOS NA DISPENSAÇÃO DE MEDICAMENTOS NO AMBIENTE HOSPITALAR
}

\author{
ERRORS IDENTIFIED IN THE DISPENSING OF MEDICINES \\ IN THE HOSPITAL ENVIRONMENT
}

Amanda Thaiz Euclides Sousa ${ }^{1}$

Leonardo Guimarães de Andrade ${ }^{2}$

RESUMO: Introdução: O Serviço de Farmácia Hospitalar tem como uma de suas atribuições essenciais estabelecer políticas e procedimentos que visem garantir um sistema de dispensação de medicamentos racional e seguro por meio de ações integradas com os diversos profissionais e gestores da saúde. Objetivo: Avaliar a ocorrência de erros na dispensação de medicamentos na unidade hospitalar. Metodologia: Trata-se de uma revisão integrativa, onde os dados formam coletados na Biblioteca Virtual em Saúde (bvs), que inclui dentre outras bases a lilacs, revistas cientificas, medline, scielo. Os critérios de inclusão foram, recorte temporal dos últimos io anos, artigos na integra e no idioma português. Como critério de exclusão, remoção os textos incompletos, artigos duplicados nas bases de dados e artigos fora da temática. Resultados: Após a análise das bibliografias, sabe-se que o tipo de sistema de distribuição de medicamentos utilizado no hospital pode influenciar na segurança da dispensação dos medicamentos. São diversas as possibilidades para que ocorra o erro na dispensação dos medicamentos, uma delas é de a prescrição médica estar errada e o atendimento de uma prescrição incorreta. Definido como a discrepância entre a ordem escrita na prescrição médica e o atendimento dessa ordem. São erros cometidos por funcionários da farmácia (farmacêuticos, inclusive) quando realizam a dispensação de medicamentos para as diversas unidades do hospital. Conclusão: Pode-se observar que os erros de dispensação de medicamentos são muito comuns nas unidades hospitalares, faz-se necessário medidas que minimizem a ocorrência deste erro, para que ocorra também a melhora na segurança do paciente e redução dos custos hospitalares.

Palavras-chaves: Medicamento. Erros de medicamento. Erros de dispensação. Hospital.

ABSTRACT: Introduction: The Hospital Pharmacy Service has as one of its essential tasks to establish policies and procedures that aim to ensure a rational and safe drug dispensing system through integrated actions with the various health professionals and managers. Objective: Assess the occurrence of errors in drug dispensing Methodology: This is an integrative review, where data are collected in the Virtual Health Library (bvs), which

${ }^{1}$ UNIG - Universidade Iguaçu. Curso de Graduação em Farmácia, Nova Iguaçu-RJ

2 Orientador. Universidade Iguaçu- UNIG .Nova Iguaçu-RJ. 
includes, among other databases, lilacs, scientific journals, medline, scielo. The inclusion criteria were, time frame of the last ro years, articles in full and in Portuguese. As an exclusion criterion, removal of incomplete texts, duplicated articles in the databases and articles outside the theme. Results: After analyzing the bibliographies, it is known that the type of drug distribution system used in the hospital can influence the safety of drug dispensing. There are several possibilities for errors in drug dispensing to occur, one of which is that the medical prescription is wrong and that the prescription is incorrect. Defined as the discrepancy between the written order in the medical prescription and the fulfillment of that order. These are mistakes made by pharmacy employees (pharmacists, including) when dispensing medication to the different units of the hospital. Conclusion: It can be observed that medication dispensing errors are very common in hospital units, it is necessary to take measures to minimize the occurrence of this error, so that there is also an improvement in patient safety and a reduction in hospital costs

Keywords: Medication. Medication errors. Dispensing errors. Hospital.

\section{INTRODUÇÃO}

Medicamento é o mesmo que fármaco, especialmente quando se encontra em uma formulação farmacêutica. Corresponde ao fármaco na especialidade farmacêutica (comprimidos, drágeas, cápsulas, soluções, pomadas etc.). (LARINI, 2008)

Segundo Barros et al, 2oro, medicamento é a droga ou a preparação com drogas de ação farmacológica benéfica, quando utilizada de acordo com as suas indicações e propriedades. Medicamentos são os produtos químicos utilizados com fim terapêutico.

A administração de medicação é uma das ações mais frequentes no ambiente hospitalar, podendo decorrer em erros, nas várias etapas do processo. O erro de medicação é considerado um evento evitável e está relacionado à prática profissional, ao produto utilizado, ao procedimento, à prescrição, aos rótulos, às embalagens, ao preparo, à dispensação, à distribuição e ao monitoramento. (TEIXEIRA, CASSIANI, 20Io)

As reações adversas a medicamentos são eventos clínicos indesejáveis atribuídos aos medicamentos utilizados em doses adequadas à enfermidade e à condição clínica do paciente. Podem provocar danos graves à saúde, ocasionando, muitas vezes, admissões hospitalares e até mesmo óbito. (MOTA, FEITAS, MACHADO, 2012)

Os eventos adversos (EA) comprometem a qualidade da atenção ao paciente hospitalizado e se referem a um dano ocorrido durante o cuidado, que não foi causado pela doença de base do paciente, sendo o uso de medicamentos um ponto crítico na assistência à saúde e ocorrência de EA. (COUTO, PEDROSA, ROSA, 20I6) 
Como a atuação farmacêutico hospitalar vai além da gestão dos medicamentos e da otimização da farmacoterapia, a qualidade e segurança da assistência permitem que o farmacêutico também contribua na redução dos erros de prescrições, de dispensação e administração de medicamentos e ainda promove sua participação nas equipes multiprofissionais (FERNANDES, 2019).

Considerando a missão da prática farmacêutica definida pela Organização Mundial de Saúde, as atividades de educação em saúde, especialmente relacionadas ao uso correto de medicamentos, a dispensação e a indicação farmacêutica podem interferir de maneira significativa no uso adequado de medicamentos pelas pessoas e pela sociedade. (ANGONESI, RENNÓ, 20II)

O Serviço de Farmácia Hospitalar tem como uma de suas atribuições essenciais estabelecer políticas e procedimentos que visem garantir um sistema de dispensação de medicamentos racional e seguro por meio de ações integradas com os diversos profissionais e gestores da saúde (SOCIEDADE BRASILEIRA DE FARMÁCIA HOSPITALAR SBRAFH, 2007).

A dispensação de medicamentos na farmácia hospitalar é citada como um dos meios de aproximar o serviço de farmácia á segurança do paciente, quanto mais eficiente o sistema de dispensação, menor será a incidência de erros e consequentemente, melhor será o serviço oferecido ao paciente (NETO, 2005).

Muitos erros de medicação são cometidos durante as diversas fases de utilização destas tecnologias no ambiente hospitalar. A dispensação é uma das fases mais sensíveis deste processo. Sistemas de dispensação seguros, organizados e efetivos são fundamentais para assegurar que cada medicamento será dispensado apropriadamente de acordo com a prescrição, reduzindo as possibilidades de erro (ANACLETO et al, 2007).

Protocolos para práticas seguras para dispensação de medicamentos foram publicados no "Protocolo para segurança na prescrição, uso e administração de medicamentos" pelo Ministério da Saúde (MS) em 2013. Segundo este documento, a farmácia deve possuir estrutura organizada e processos de trabalho escritos e difundidos para promover a prevenção, identificação e redução de erros de prescrição e dispensação (BRASIL, 2013b). 


\section{OBJETIVOS}

\section{I Objetivo geral} hospitalar

Avaliar a ocorrência de erros na dispensação de medicamentos no ambiente

\subsection{Objetivos Específicos}

I. Avaliar os tipos de erros de dispensação de medicamentos

2. Caracterizar os fatores envolvidos na ocorrência dos erros

3. Criação dos sistemas operacionais que minimizem os erros na dispensação de medicamentos

4. Elaboração de um fluxograma que permita a diminuição dos erros

\section{JUSTIFICATIVA}

O presente estudo foi motivado pela importância do tema, de se descartar os medicamentos de forma segura para que haja segurança de todos os que manipulam e evitar eventos adversos. Espera-se que o conhecimento da frequência de erros e das condições predisponentes para sua ocorrência, forneça informações que possibilitem sua correção e diminuição do erro de dispensação.

\section{METOdOlOGia}

O estudo se utilizará do método de revisão integrativa da literatura, que tem como finalidade reunir, e resumir o conhecimento científico, antes produzido sobre o tema investigado. Avalia, sintetiza e busca nas evidências disponíveis a contribuição para o desenvolvimento da temática (MENDES, SILVEIRA, GALVÃO, 2008).

Os dados foram coletados em artigos nacionais e internacionais obtidos via internet em periódicos indexados em bases de dados eletrônicas tais como: Scielo (ScientificElectronic Library Online), Pubmed (Literatura Médica Internacional), Lilacs (Literatura Latino-Americano e do Caribe em Ciência da Saúde), revistas cientificas, MEDLINE (Literatura Internacional em Ciências Da Saúde). O período de pesquisa ocorreu no ano de 202I, com associação de descritores: "erros de dispensação", "hospitalar", "farmácia". Como critério de inclusão, fora utilizado o recorte temporal dos últimos io anos, 
artigos com texto disponibilizados na íntegra e no idioma português. Como critérios de exclusão, consideraram-se para a remoção os textos incompletos, artigos duplicados nas bases de dados e artigos fora da temática.

A pesquisa resultou em ro artigos distintos distribuídos em mais de uma base de dados, reunindo estudos de revisão ou apresentação de dados primários referente a erros de dispensação.

\section{DISCUSSÃO}

Nos últimos anos a pesquisa sobre erros de dispensação em hospitais no Brasil têm crescido.

Para a discussão, é importante considerar que, embora a pesquisa sobre os erros de dispensação no Brasil venha ganhando espaço nos últimos anos, a literatura a respeito do tema ainda é escassa. (SIRTOLI et al, 2018)

Sabe-se que o tipo de sistema de distribuição de medicamentos utilizado no hospital pode influenciar na segurança da dispensação dos medicamentos. (COSTA, VALLI, ALVARENGA, 2010)

São diversas as possibilidades para que ocorra o erro na dispensação dos medicamentos, uma delas é de a prescrição médica estar errada e o atendimento de uma prescrição incorreta. Definido como a discrepância entre a ordem escrita na prescrição médica e o atendimento dessa ordem. São erros cometidos por funcionários da farmácia (farmacêuticos, inclusive) quando realizam a dispensação de medicamentos para as diversas unidades do hospital. (ANACLETO et al, 2010)

Definido como a discrepância entre a ordem escrita na prescrição médica e o atendimento dessa ordem, pode ser classificado em erros de conteúdo, erros de rotulagem e erros de documentação. (ANACLETO et al, 2010).

- Erros de conteúdo: são aqueles referentes ao conteúdo da dispensação, ou seja, relacionados aos medicamentos que estão prescritos e serão dispensados.

- Erros de rotulagem: são os erros relacionados aos rótulos dos medicamentos dispensados que podem gerar dúvidas no momento da dispensação e/ou administração, erros de grafia nos rótulos e tamanho de letras que impedem a leitura, a identificação ou 
podem levar ao uso incorreto do medicamento. São considerados os rótulos do próprio produto, as etiquetas impressas na farmácia e utilizadas na identificação dos medicamentos, das misturas intravenosas e da nutrição parenteral preparadas na farmácia. Pode aparecer como nome do paciente errado, nome do medicamento errado, concentração errada do medicamento, forma farmacêutica errada, quantidade errada, data errada, orientações erradas relacionadas ao uso ou armazenamento.

- Erros de documentação: são os erros relacionados à documentação de registro do processo de dispensação, como por exemplo, a ausência ou registro incorreto da dispensação de medicamentos controlados, falta de data na prescrição, falta de assinatura do prescritor ou do dispensador, dentre outros.

Alguns estudos mostram as taxas de erros de dispensação, como uso da leitura em código de barras, observa-se que medicamentos são dispensados contendo algum tipo de erros, as doses de medicamentos devem ser conferidas pelo farmacêutico antes da dispensação. (JAYME, CARNEIRO, 2016)

Segundo ISMP, 2020, para a classificação dos erros de dispensação de medicamentos, os seguintes domínios podem ser considerados:

I. Omissão de medicamento: o medicamento prescrito não foi dispensado;

2. Omissão de dose: o número de doses dispensadas é menor que a prescrita;

3. Adição de dose: o número de doses dispensadas é maior que a prescrita;

4. A embalagem: medicamentos dispensados com identificação errada do paciente;

5. Concentração errada: medicamento dispensado com concentração inferior ou superior à prescrita;

6. Forma farmacêutica errada: medicamento dispensado com forma farmacêutica diferente da prescrita;

7. Desvio de qualidade: medicamento dispensado com erros de rotulagem ou desvio de qualidade;

8. Erro no horário da dispensação: medicamento dispensado em horário errado;

9. Medicamento errado I: um medicamento estava prescrito, mas outro foi dispensado;

10. Medicamento errado 2: um medicamento não prescrito foi dispensado, além do prescrito. 
Os erros de dispensação demonstram fragilidades nos processos de trabalho e elevados custos para o setor de saúde em função de promoverem a necessidade de execução produtividade do indivíduo, sofrimento e redução na expectativa

de vida. Esses eventos afetam, ainda, a credibilidade dos pacientes para com o sistema e os profissionais de saúde. (GALVÃO et al, 2012).

FIGURA I - Fluxograma do processo de dispensação dos medicamentos prescritos.
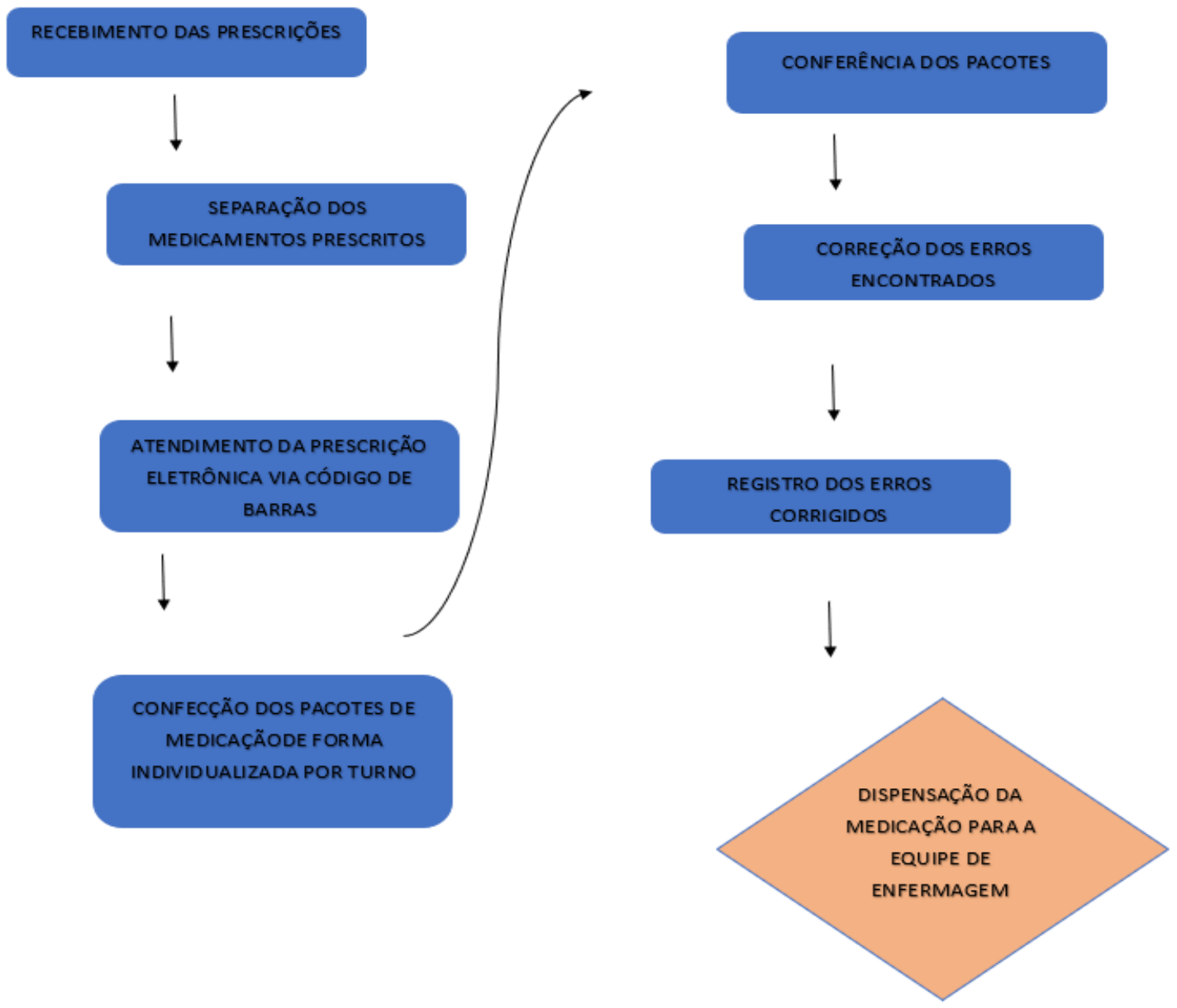

Fonte: Adaptado pelo autor, 2021

Diante disso, sistemas de dispensação de medicamentos seguros, organizados e eficientes são essenciais para controlar os custos e garantir que a prescrição médica seja seguida com segurança, conforme solicitado e no prazo apropriado, contribuindo para 
minimizar a ocorrência de erros de dispensação de medicamentos em serviços de saúde. (JACOBSEN, 2015)

As consequências dos erros de dispensação podem ser significativas tais como o alto custo para o sistema de saúde, a alteração dos resultados terapêuticos esperados para os pacientes e o aumento morbimortalidade, além de problemas psicológicos para os profissionais de saúde envolvidos nos processos. (VILELA, 2019)

\section{CONCLUSÃO}

Pode-se observar que os erros de dispensação de medicamentos são muito comuns nas unidades hospitalares, faz-se necessário medidas que minimizem a ocorrência deste erro, para que ocorra também a melhora na segurança do paciente e redução dos custos hospitalares. O estudo possui grande importância científica para os profissionais da saúde e as instituições hospitalares pois amplia os conhecimentos a cerca de um assunto tão importante.

\section{REFERÊNCIAS BIBLIOGRÁFICAS}

ANGONESI, D.; RENNÓ, M. U. P. Dispensação farmacêutica: proposta de um modelo para a prática. Ciência \& Saúde Coletiva [online]. 20II, v. I6, n.9 pp. 3883-3891. Disponível em: 〈https://doi.org/10.1590/Si413-81232011001000024〉.

ANACLETO, T.A.; PERINI, E.; ROSA, M.B. et al. Drug-dispensing errors in the hospital pharmacy. Clinics. v. 62, n. 3, p. 243-250, 2007.

ANACLETO, T.A.; ROSA, M. B, NEIVA, H.M. et al. Farmácia Hospitalar. Erros de Medicação (Encarte) .Pharm Bras. Janeiro/Fevereiro 2010.

BARROS, E. Et al. Medicamentos na prática clínica. Artmed EditoraS.A.2oro.

COSTA, L.A.; VALLI, C.; ALVARENGA, A.P. Medication dispensing errors at a public pediatric hospital. Rev Latino-am Enfermagem. 2010;16(5):812-7.

COUTO R.C, PEDROSA, T.M.G, ROSA, M.B. Erros acontecem. A força da transparência para o enfrentamento dos eventos adversos assistenciais em pacientes hospitalizados. Construindo um sistema de saúde mais seguro. Instituto de Estudos de Saúde Suplementar . Universidade Federal de Minas Gerais. 2016.

FERNANDES, L.L. (2019). A importância do farmacêutico hospitalar juntamente com a 
equipe multidisciplinar na Unidade de Terapia Intensiva (UTI). Revista Farol, 8(8), 5-2I.

GALVÃO, A.A.; et al. Identificação dos erros de dispensação em uma farmácia hospitalar: um estudo comparativo no município de Salvador Bahia; R. Ci. med. biol. Salvador 2012;II(I): 201-206.

ISMP [Internet]. Boletim [citado em 26 março de 2020]. Disponível em: https://www.ismp-brasil.org/site/boletins/

JACOBSEN, T.F.; MUSSI, M.M.; TELIS, M.P.; Análise de erros de prescrição em um hospital da região sul do Brasil. Rev. Bras. Farm. Hosp. Serv. Saúde São Paulo v.6 n.3 23-26 jul./set. 2015 .

JAYME, M. O.; CARNEIRO, M. B. Tecnologia de código de barras e a prevenção de erros na dispensação de medicamentos. Rev. Bras. Farm. Hosp. Serv. Saúde. São Paulo, v. 7, n. 2, p. 22-25, jun. 2016.

LARINI, LOURIVAL. Fármacos e medicamentos (recurso eletrônico)- dados eletrônicosPorto Alegre: Artmed, 2008.

MENDES, K. DAL S.; SILVEIRA, R. C. DE C. P E.; GALVÃO, C. M. Revisão integrativa: método de pesquisa para a incorporação de evidências na saúde e na enfermagem. Texto \& Contexto - Enfermagem [online]. 2008, v. 17, n. 4

MINISTÉRIO DA SAÚDE. Portaria no . 529 de Io. de abril de 2013. Institui o Programa Nacional de Segurança do Paciente. Brasília: Ministério da Saúde, 2orzb.

Mota DM, Melo JRR, Freitas DRC, Machado M. Perfil da mortalidade por intoxicação com medicamentos no Brasil, 1996-2005: retrato de uma década. Ciênc Saúde Colet 2012; 17:61-70.

NETO, J.F.M. Farmacia Hospitalar e suas interfaces com a saúde. São Paulo, SP.i ed. RX, 2005

Organización Pan-Americana de la Salud (OPAS)/ Organización Mundial de la Salud (OMS). El papel del farmacéutico en la atencíon a salud. Informe de la reunión de la OMS, Tokio, Japon, 3I ago. al 3 sep. de I993. Washington: PAHO; 1995.

SIRTOLI R, P. E.C.P.; FIEIRA, C.; VOLKWEIS, J.G.; PENTEADO, S.T.S.; CALDEIRA, L.F.C. Perfil e análise de pareto dos erros de dispensação de um hospital público. Rev. Bras. Farm. Hosp. Serv. Saúde, 9(2): pag-pag, 2019. Doi: 10.30968/rbfhss.2018.092.007

SOCIEDADE BRASILEIRA DE FARMÁCIA HOSPITALAR. SBRAFH. Padrões mínimos para Farmácia Hospitalar, Goiânia, 2007, 20p. 
TEIXEIRA, T.C.A.; CASSIANI, S.H.B.; Análise de causa raiz: avaliação de erros de medicação em um hospital universitário. Rev esc enferm USP. 2010;44(I):139-46.

VILELA, R.P.; JERICÓ, M.C. Implementing technologies to prevent medication erros at a high-complexity hospital: analysis of cost and results. einstein (São Paulo). 2019;17(4):eGS462I. http://dx.doi.org/ Io.31744/einstein_journal/2019GS462I 